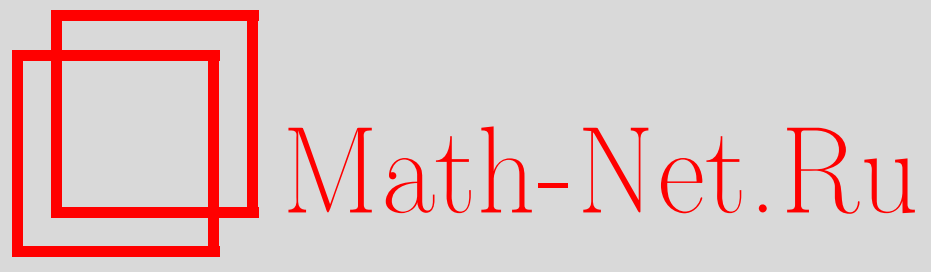

М. В. Демина, Н. А. Кудряшов, Специальные полиномы и рациональные решения иерархии второго уравнения Пенлеве, ТМФ, 2007, том 153, номер 1, 58-67

DOI: https://doi.org/10.4213/tmf6121

Использование Общероссийского математического портала Math-Net.Ru подразумевает, что вы прочитали и согласны с пользовательским соглашением http: //www. mathnet.ru/rus/agreement

Параметры загрузки:

IP : 18.207 .199 .55

26 апреля 2023 г., 15:27:50 


\title{
СПЕЦИАЛЬНЫЕ ПОЛИНОМЫ И РАЦИОНАЛЬНЫЕ РЕШЕНИЯ ИЕРАРХИИ ВТОРОГО УРАВНЕНИЯ ПЕНЛЕВЕ
}

\begin{abstract}
Изучены специальные полиномы, используемые для представления рациональных решений иерархии второго уравнения Пенлеве. Найден ряд рекуррентных соотношений, которому удовлетворяют эти полиномы. В частности, получено дифференциально-разностное соотношение, позволяющее рекуррентно находить любой полином. Это соотношение является аналогом уравнений для цепочки Тоды.
\end{abstract}

Ключевые слова: уравнения Пенлеве, иерархия второго уравнения Пенлеве, рациональные решения, специальные полиномы, цепочка Тоды.

\section{1. ВВЕДЕНИЕ}

Хорошо известно, что общие решения шести уравнений Пенлеве $P_{1}-P_{6}$ не выражаются через элементарные или специальные функции, т.е. определяют новые трансцендентные функции, называемые трансцендентами Пенлеве [1]-[3]. Однако пять из шести уравнений (уравнения $P_{2}-P_{6}$ ) имеют семейства рациональных или алгебраических решений при определенных значениях параметров. Как правило, для описания таких решений используются семейства специальных полиномов.

Яблонский [4] и Воробьев [5] предложили представление через полиномы для рациональных решений второго уравнения Пенлеве $P_{2}$

$$
w_{z z}=2 w^{3}+z w+\alpha .
$$

Они показали, что $P_{2}$ имеет единственное рациональное решение при любом целом значении параметра $\alpha$, причем совокупность таких решений выражается через логарифмическую производную некоторых полиномов, получивших название полиномов Яблонского-Воробьева [6]. Специальные полиномы, связанные с определенными рациональными и алгебраическими решениями других уравнений Пенлеве, были предложены Окамото [7] и Умемурой [8].

* Московский инженерно-физический институт (государственный университет), Москва, Россия. E-mail: mvdemina@mephi.ru,kudryashov@mephi.ru 
Заметим, что уравнения $P_{2}-P_{6}$ также имеют однопараметрические семейства решений, выражаемые через классические специальные функции. Некоторые ортогональные многочлены возникают как частный случай таких решений и, следовательно, порождают рациональные решения рассматриваемых уравнений. Например, для $P_{3}$ и $P_{5}$ такими многочленами являются полиномы Лагерра, а для $P_{4}$ и $P_{6}-$ полиномы Эрмита и Якоби, соответственно.

В последнее время изучению специальных полиномов, связанных с уравнениями Пенлеве, уделяется большое внимание. Полиномы Яблонского-Воробьева, Окамото и Умемуры рассматривают как аналоги классических ортогональных многочленов. Эти полиномы обладают целым рядом интересных свойств. В частности, они выражаются через полиномы Шура и удовлетворяют обыкновенным дифференциальным уравнениям. Кроме того, их корни на комплексной плоскости имеют ярко выраженную регулярную структуру. Также они непосредственно связаны с так называемой $\tau$-функцией уравнений Пенлеве. Отметим, что эти семейства полиномов имеют большое количество физических приложений. Например, через них могут быть выражены частные решения многих известных нелинейных эволюционных уравнений. Так, через полиномы Яблонского-Воробьева выражаются решения уравнения Кортевега-де Фриза, уравнения Кадомцева-Петвиашвили, нелинейного уравнения Шредингера [6] и т.д.

Данная работа посвящена изучению рациональных решений и связанных с ними специальных полиномов целой иерархии обыкновенных дифференциальных уравнений, первым представителем которой является $P_{2} \equiv P_{2}^{(1)}$. Эта иерархия является редукцией иерархии модифицированного уравнения Кортевега-де Фриза. Она выглядит следующим образом [3], [9]:

$$
P_{2}^{(N)}[w, \alpha]:\left(\frac{d}{d z}+2 w\right) L_{N}\left[w_{z}-w^{2}\right]-z w-\alpha=0, \quad N \geqslant 1,
$$

где $L_{N}\left[w_{z}-w^{2}\right] \equiv L_{N}[u]$ задается соотношением Ленарда

$$
d_{z} L_{N+1}[u]=\left(d_{z}^{3}+4 u d_{z}+2 u_{z}\right) L_{N}[u], \quad L_{0}[u]=\frac{1}{2} .
$$

Как и $P_{2}$, уравнения иерархии имеют единственное рациональное решение при каждом целом $\alpha=n \in \mathbb{Z}$. Эти решения можно записать в виде

$$
w^{(N)}(z ; n)=\frac{d}{d z}\left\{\ln \frac{Q_{n-1}^{(N)}(z)}{Q_{n}^{(N)}(z)}\right\}, \quad w^{(N)}(z ;-n)=-w^{(N)}(z ; n),
$$

где $n \geqslant 1$, а $\left\{Q_{n}^{(N)}(z)\right\}$ - семейство специальных полиномов, которые при любом $N$ мы будем называть полиномами Яблонского-Воробьева, учитывая, что каждое $N$ задает свое семейство полиномов. Очевидно, что первое семейство (при $N=1$ ) образуют именно те полиномы, которые были введены Яблонским и Воробьевым для $P_{2}$. Старшие семейства во многом аналогичны $\left\{Q_{n}^{(1)}(z)\right\}$. Например, они также выражаются через полиномы Шура. Не так давно Кларксон и Мэнсфилд изучали расположение корней этих полиномов на комплексной плоскости [10]. В работе [11] 
были найдены формулы для степеней и коэффициентов любого семейства полиномов. Там же было показано, что так называемые симметрические функции корней связаны со степеннь́м разложением решений уравнений (1.2) в окрестности бесконечности.

Основным результатом данной работы является вывод дифференциально-разностного соотношения

$$
Q_{n+1}^{(N)} Q_{n-1}^{(N)}=z\left(Q_{n}^{(N)}\right)^{2}-2\left(Q_{n}^{(N)}\right)^{2} L_{N}\left[2 \frac{d^{2}}{d z^{2}} \ln Q_{n}^{(N)}\right], \quad n \geqslant 1,
$$

позволяющего находить полиномы любого семейства.

В разделе 2 найдено несколько рекуррентных соотношений, которым удовлетворяют полиномы $\left\{Q_{n}^{(N)}(z)\right\}$, в частности, (1.5); в разделе 3 рассмотрены первые несколько семейств полиномов.

\section{2. РЕКУРРЕНТНЫЕ ФОРМУЛЫ ДЛЯ ПОЛИНОМОВ ЯБЛОНСКОГО-ВОРОБЬЕВА}

Яблонский и Воробьев вывели дифференциально-разностное соотношение, которое может быть использовано для построения специальных полиномов, связанных с $P_{2}$, т.е. семейства $\left\{Q_{n}^{(1)}(z)\right\}$ [4], [5]. Оно имеет вид

$$
Q_{n+1}^{(1)} Q_{n-1}^{(1)}=z\left(Q_{n}^{(1)}\right)^{2}-4\left[Q_{n}^{(1)}\left(Q_{n}^{(1)}\right)^{\prime \prime}-\left(Q_{n}^{(1)}\right)^{\prime}\left(Q_{n}^{(1)}\right)^{\prime}\right]
$$

где $Q_{0}^{(1)}(z)=1, Q_{1}^{(1)}(z)=z$. Далеко не очевидно, что (2.1) определяет действительно полиномы, а не рациональную функцию, поскольку на каждом шаге для нахождения $Q_{n+1}^{(1)}(z)$ приходится делить правую часть $(2.1)$ на $Q_{n-1}^{(1)}(z)$. То, что соотношение задает именно полиномы - это действительно замечательный факт. Выведем соотношение, аналогичное (2.1), для любого семейства $\left\{Q_{n}^{(N)}(z)\right\}$. Для этого нам потребуются некоторые дополнительные рекуррентные формулы.

Теорема 1. Любое семейство полиномов Яблонского-Воробъева удовлетворяет следующим рекуррентным соотношениям:

$$
\begin{aligned}
D_{z} Q_{n+1}^{(N)} \cdot Q_{n-1}^{(N)} & =(2 n+1)\left(Q_{n}^{(N)}\right)^{2}, \\
D_{z}^{2} Q_{n+1}^{(N)} \cdot Q_{n}^{(N)} & =0 .
\end{aligned}
$$

Здесъ $D_{z}$ - это оператор Xиротъ,

$$
D_{z} f(z) \cdot g(z)=\left.\left(\frac{d}{d z_{1}}-\frac{d}{d z_{2}}\right)\left(f\left(z_{1}\right) g\left(z_{2}\right)\right)\right|_{z_{1}=z_{2}=z}
$$

ДоКАЗАТЕЛЬСТво. Для вывода соотношений (2.2) и (2.3) воспользуемся иерархией уравнений Шварца- $P_{2}[12]$, которую можно записать в виде

$$
2 \psi_{z} L_{N}\left[\frac{1}{2}\left(\frac{\psi_{z z}}{\psi_{z}}\right)_{z}-\frac{1}{4}\left(\frac{\psi_{z z}}{\psi_{z}}\right)^{2}\right]+(1-2 \alpha) \psi-z \psi_{z}=0 .
$$


Пусть $\psi^{(N)}(z ; \alpha) \equiv \psi_{\alpha}$ и $w^{(N)}(z ; \alpha) \equiv w_{\alpha}$ - решения $(2.5)$ и $(1.2)$, соответственно. Тогда преобразование

$$
w_{\alpha}=\frac{1}{2} \frac{d}{d z} \ln \psi_{\alpha}^{\prime},
$$

где штрих означает производную по $z$, осуществляет отображение $N$-го уравнения иерархии Шварца- $P_{2}$ на $N$-е уравнение иерархии $P_{2}$. Далее, зафиксировав $N$, не будем писать верхний индекс. Пусть $\alpha=n \in \mathbb{Z}$, тогда рациональным решениям $\psi_{n}$ уравнения (2.5) соответствуют рациональные решения $w_{n}$ уравнения (1.2) [12]. Полагая $\alpha=n \geqslant 1$ и сравнивая (1.4), (2.6), мы находим связь между двумя соседними полиномами $Q_{n-1}, Q_{n}$ и функцией $\psi_{n}^{\prime}$ :

$$
\left(\frac{Q_{n-1}(z)}{Q_{n}(z)}\right)^{2}=a(n) \psi_{n}^{\prime} .
$$

Отсюда немедленно следует равенство

$$
a(n) \psi_{n}=\int^{z}\left(\frac{Q_{n-1}(s)}{Q_{n}(s)}\right)^{2} d s,
$$

где путь интегрирования не проходит через полюсы подынтегральной функции. Два решения $\psi_{\alpha}$ и $\psi_{\alpha-1}$ уравнения $(2.5)$ связаны итерационной формулой

$$
\psi_{\alpha}^{\prime}=\frac{\psi_{\alpha+1}^{2}}{\psi_{\alpha+1}^{\prime}} .
$$

Пусть $\alpha=n \geqslant 1$; тогда, подставляя (2.7), (2.8) в это соотношение, получаем

$$
\frac{Q_{n-1}(z)}{Q_{n+1}(z)}=b(n) \int^{z}\left(\frac{Q_{n}(s)}{Q_{n+1}(s)}\right)^{2} d s .
$$

Дифференцируя это равенство по $z$ и умножая результат на $Q_{n+1}^{2}$, мы находим, что

$$
Q_{n+1}^{\prime} Q_{n-1}-Q_{n+1} Q_{n-1}^{\prime}=c(n) Q_{n}^{2} .
$$

Константа $c(n)$ может быть произвольной, поскольку рациональное решение $w_{n}$ инвариантно относительно преобразования $Q_{n} \rightarrow e(n) Q_{n}$. Яблонский и Воробьев рассматривали приведенные полиномы, поэтому выберем $c(n)$ так, чтобы полиномы были приведенными. Поскольку степень $Q_{n}$ равна $n(n+1) / 2$ и не зависит от $N$ [11], положим $c(n)=2 n+1$. Таким образом, справедливость соотношения (2.2) доказана. Перейдем к выводу соотношения (2.3).

Обозначим кратность корня $z=a$ полинома $Q_{n}(z)$ через $d_{n}(a)$. Если точка $z=a$ не является корнем $Q_{n}(z)$, то считаем, что $d_{n}(a)=0$. Известно, что рациональные решения уравнения (1.2) могут иметь только простые полюсы с вычетами, равными $k$ или $-k, 0<k \leqslant N$. Покажем, что для любого рационального решения $w_{n}$, имеющего в некоторой точке $z=a$ полюс с вычетом $k(-k)$ выполнены неравенства

$$
d_{n-1}(a) \geqslant \frac{k(k+1)}{2}, \quad d_{n}(a) \geqslant \frac{k(k-1)}{2}
$$




$$
\left(d_{n-1}(a) \geqslant \frac{k(k-1)}{2}, \quad d_{n}(a) \geqslant \frac{k(k+1)}{2}\right) .
$$

Применим метод математической индукции. При $k=1$ это утверждение справедливо. Пусть оно также справедливо при $k=m-1$, т.е. для каждого рационального решения, которое имеет в некоторой точке полюс с вычетом $m-1(1-m)$, выполнены соответствующие неравенства. Докажем, что тогда утверждение верно при $k=m$. Будем доказывать от противного, т.е. предположим, что некоторое $w_{n}$ имеет полюс вычета $m(-m)$ в точке $z=a$, но

$$
\begin{array}{rlrl}
d_{n-1}(a)<\frac{m(m+1)}{2}, & & d_{n}(a)<\frac{m(m-1)}{2} \\
\left(d_{n-1}(a)<\frac{m(m-1)}{2},\right. & \left.d_{n}(a)<\frac{m(m+1)}{2}\right) .
\end{array}
$$

При этом, очевидно, должно выполняться равенство

$$
d_{n-1}(a)-d_{n}(a)=m \quad\left(d_{n}(a)-d_{n-1}(a)=m\right) .
$$

Перепишем соотношение (2.2) в интегральном виде:

$$
\begin{aligned}
Q_{n+1} & =(2 n+1) Q_{n-1} \int^{z}\left(\frac{Q_{n}(s)}{Q_{n-1}(s)}\right)^{2} d s, \\
Q_{n-1} & =-(2 n+1) Q_{n+1} \int^{z}\left(\frac{Q_{n}(s)}{Q_{n+1}(s)}\right)^{2} d s .
\end{aligned}
$$

Не ограничивая общности, рассмотрим случай положительного вычета. Используя $(2.12)$, найдем $d_{n+1}(a)$. Имеем $d_{n+1}(a)=d_{n-1}(a)+1-2 m$. Тогда рациональное решение $w_{n+1}$ также имеет полюс в точке $z=a$ с вычетом $d_{n}(a)-d_{n+1}(a)=$ $d_{n}(a)-d_{n-1}(a)-1+2 m=-m-1+2 m=m-1$. Но при этом

$$
d_{n}(a)<\frac{m(m-1)}{2}, \quad d_{n+1}(a)<\frac{m(m+1)}{2}+1-2 m=\frac{(m-1)(m-2)}{2},
$$

что противоречит предположению индукции. В случае отрицательного вычета, используя соотношение (2.13), также приходим к противоречию.

Любое рациональное решение $w_{n}$ можно представить в виде отношения двух полиномов $g(z)$ и $f(z)$, т.е. $w_{n}=g(z) / f(z)$, где $g(z), f(z)$ удовлетворяют системе двух уравнений [13]

$$
\begin{aligned}
& f^{\prime \prime} f-\left(f^{\prime}\right)^{2}+g^{2}=0 \\
& \left(\frac{d}{d z}+2 \frac{g}{f}\right) L_{N}\left[\frac{g^{\prime}}{f}-\frac{g\left(f^{\prime}+g\right)}{f^{2}}\right]-z \frac{g}{f}-n=0 .
\end{aligned}
$$

Причем, если $w_{n}$ имеет $l_{i}$ полюсов с вычетом, равным $i$, в точках $z=z_{n, j}^{(i)}\left(1 \leqslant j \leqslant l_{i}\right.$, $-k \leqslant i \leqslant k, i \neq 0)$, то

$$
f(z)=\prod_{\substack{i=-k, j=1 \\ i \neq 0}}^{k} \prod_{i}^{l_{i}}\left(z-z_{n, j}^{(i)}\right)^{i^{2}}
$$


В силу доказанного произведение полиномов $Q_{n-1}$ и $Q_{n}$ устроено следующим обра3ом:

$$
Q_{n-1}(z) Q_{n}(z)=\prod_{\substack{i=-k, j=1 \\ i \neq 0}}^{k} \prod_{i}^{l_{i}}\left(z-z_{n, j}^{(i)}\right)^{i^{2}} h(z) \equiv f(z) h(z)
$$

где $h(z)$ - некоторый полином. Степень полинома $f(z)$ найдена в [13]:

$$
\operatorname{deg}(f(z)) \equiv \sum_{\substack{i=-k \\ i \neq 0}}^{k} l_{i} i^{2}=n^{2}
$$

Отсюда мы видим, что $\operatorname{deg}\left(Q_{n-1} Q_{n}\right)=\operatorname{deg}(f)$. Следовательно, $h(z) \equiv 1$. Подставляя $f=Q_{n-1} Q_{n}, g=Q_{n-1}^{\prime} Q_{n}-Q_{n-1} Q_{n}^{\prime}$ в первое уравнение системы $(2.14)$, находим искомое соотношение $(2.3)$.

ЗАмечаниЕ. Соотношения (2.2), (2.3) при $N=1$ известны [5], [10]; при $N>1$ они получены впервые.

СлЕДСТВИЕ 1. Еще одно дифференииально-разностное соотношение для полиномов $\left\{Q_{n}^{(N)}(z)\right\}$ может быть получено подстановкой $f=Q_{n-1} Q_{n}, g=Q_{n-1}^{\prime} Q_{n}-$ $Q_{n-1} Q_{n}^{\prime}$ во второе уравнение системь (2.14).

СлЕДСтвиЕ 2. В процессе доказательства теоремы было также показано, что соседние полиномы $Q_{n-1}$ и $Q_{n}$ не имеют корней, отсутствующих в рациональном решении $w_{n}$, т.е. не являющихся полюсами $w_{n}$. Другими словами, $Q_{n-1}$ и $Q_{n}$ не имеют совпадающих корней одинаковой кратности. Более того, любой полюс $w_{n}$ вычета, равного $k(-k)$, одновременно является корнем полиномов $Q_{n-1}, Q_{n}$ кратности $k(k+1) / 2, k(k-1) / 2(k(k-1) / 2, k(k+1) / 2)$, соответственно.

Соотношения (2.2), (2.3) не могут быть прямо использованы для рекуррентного построения полиномов. Даже (2.2), переписанное в интегральном виде, не годится для этой цели, поскольку не определена константа интегрирования. Тем не менее с помощью (2.2), (2.3) такое соотношение может быть найдено.

Преобразование Беклунда для решений рассматриваемой иерархии имеет вид [14], $[15]$

$$
w(z ; \alpha+1)=-w(z ; \alpha)+\frac{2 \alpha+1}{2 L_{N}\left[-w_{z}(z ; \alpha)-w^{2}(z ; \alpha)\right]-z}, \quad \alpha \neq-\frac{1}{2}
$$

(мы опять не пишем верхний индекс $N$ ). Номер уравнения фиксируется оператором Ленарда. Нетрудно показать, что для любого уравнения иерархии при $\alpha=n \geqslant 1$ справедливы равенства

$$
\begin{aligned}
w_{n}+w_{n+1} & =-\frac{D_{z} Q_{n+1} \cdot Q_{n-1}}{Q_{n+1} Q_{n-1}} \\
-\left(w_{n}^{\prime}+w_{n}^{2}\right) & =2 \frac{d^{2}}{d z^{2}}\left(\ln Q_{n}\right)-\frac{D_{z}^{2} Q_{n} \cdot Q_{n-1}}{Q_{n} Q_{n-1}} .
\end{aligned}
$$


Полагая $\alpha=n \geqslant 1$ в (2.18) и используя (2.19) и теорему 1 , получаем

$$
Q_{n+1}^{(N)} Q_{n-1}^{(N)}=z\left(Q_{n}^{(N)}\right)^{2}-2\left(Q_{n}^{(N)}\right)^{2} L_{N}\left[2 \frac{d^{2}}{d z^{2}}\left(\ln Q_{n}^{(N)}\right)\right] .
$$

Это и есть дифференциально-разностное соотношение, которое, если его дополнить условиями $Q_{0}^{(N)}(z)=1, Q_{1}^{(N)}(z)=z$, позволяет последовательно находить любой полином по двум предыдущим.

\section{3. НЕКОТОРЫЕ ПРИМЕРЫ}

В этом разделе мы получим несколько полиномов из четырех первых семейств $\left\{Q_{n}^{(N)}(z)\right\}, N=1,2,3,4$. Используя рекуррентную формулу Ленарда (1.3), находим

$$
\begin{aligned}
& L_{1}[u]=u \\
& L_{2}[u]=u_{z z}+3 u^{2} \\
& L_{3}[u]=u_{z z z z}+10 u u_{z z}+5 u_{z}^{2}+10 u^{3} \\
& L_{4}[u]=u_{z z z z z z}+14 u u_{z z z z}+28 u_{z} u_{z z z}+21 u_{z z}^{2}+70 u^{2} u_{z z}+70 u u_{z}^{2}+35 u^{4} .
\end{aligned}
$$

Соотношение (2.20) при $N=1$ совпадает с (2.1). При $N=2$ это соотношение было найдено в работе [16]. Оно имеет вид

$$
Q_{n+1}^{(2)} Q_{n-1}^{(2)}=z\left(Q_{n}^{(2)}\right)^{2}-4\left[Q_{n}^{(2)}\left(Q_{n}^{(2)}\right)^{\prime \prime \prime \prime}-4\left(Q_{n}^{(2)}\right)^{\prime \prime \prime}\left(Q_{n}^{(2)}\right)^{\prime}+3\left(Q_{n}^{(2)}\right)^{\prime \prime}\left(Q_{n}^{(2)}\right)^{\prime \prime}\right] .
$$

Заметим, что (2.1), (3.5) можно переписать следующим образом:

$$
\begin{aligned}
& Q_{n+1}^{(1)} Q_{n-1}^{(1)}=z\left(Q_{n}^{(1)}\right)^{2}-2 D_{z}^{2} Q_{n}^{(1)} \cdot Q_{n}^{(1)}, \\
& Q_{n+1}^{(2)} Q_{n-1}^{(2)}=z\left(Q_{n}^{(2)}\right)^{2}-2 D_{z}^{4} Q_{n}^{(2)} \cdot Q_{n}^{(2)} .
\end{aligned}
$$

Полиномы Яблонского-Воробьева $Q_{n}^{(1)}(z)$ имеют вид

$$
\begin{aligned}
Q_{2}^{(1)}(z) & =z^{3}+4, \\
Q_{3}^{(1)}(z) & =z^{6}+20 z^{3}-80, \\
Q_{4}^{(1)}(z) & =\left(z^{9}+60 z^{6}+11200\right) z \\
Q_{5}^{(1)}(z) & =z^{15}+140 z^{12}+2800 z^{9}+78400 z^{6}-313600 z^{3}-6272000, \\
Q_{6}^{(1)}(z) & =z^{21}+280 z^{18}+18480 z^{15}+627200 z^{12}-17248000 z^{9}+1448832000 z^{6}+ \\
& +19317760000 z^{3}-38635520000 .
\end{aligned}
$$

Несколько первых полиномов семейства $\left\{Q_{n}^{(2)}(z)\right\}$ представлены ниже:

$$
\begin{aligned}
& Q_{2}^{(2)}(z)=z^{3} \\
& Q_{3}^{(2)}(z)=z\left(z^{5}-144\right) \\
& Q_{4}^{(2)}(z)=z^{10}-1008 z^{5}-48384
\end{aligned}
$$




$$
\begin{aligned}
& Q_{5}^{(2)}(z)=z^{15}-4032 z^{10}-3048192 z^{5}+146313216 \\
& Q_{6}^{(2)}(z)=z\left(z^{20}-12096 z^{15}-21337344 z^{10}-33798352896 z^{5}-4866962817024\right) .
\end{aligned}
$$

Что касается старших семейств полиномов, формула (2.20), разрешенная относительно $Q_{n+1}^{(N)}$, содержит в знаменателе не только $Q_{n-1}^{(N)}$, но и $\left(Q_{n}^{(N)}\right)^{k}$, где $k-$ натуральное число, зависящее от $N$. Однако согласно доказанному выше соотношение (2.20) при любом $N$ определяет полиномы. Несколько первых полиномов семейств $\left\{Q_{n}^{(3)}(z)\right\},\left\{Q_{n}^{(4)}(z)\right\}$ имеют следующий вид.

Полиномы Яблонского-Воробьева $Q_{n}^{(3)}(z)$ :

$$
\begin{aligned}
& Q_{2}^{(3)}(z)=z^{3} \\
& Q_{3}^{(3)}(z)=z^{6} \\
& Q_{4}^{(3)}(z)=z^{3}\left(z^{7}+14400\right), \\
& Q_{5}^{(3)}(z)=z\left(z^{14}+129600 z^{7}-373248000\right), \\
& Q_{6}^{(3)}(z)=z^{21}+648000 z^{14}-24634368000 z^{7}-35473489920000
\end{aligned}
$$

полиномы Яблонского-Воробьева $Q_{n}^{(4)}(z)$ :

$$
\begin{aligned}
& Q_{2}^{(4)}(z)=z^{3}, \\
& Q_{3}^{(4)}(z)=z^{6}, \\
& Q_{4}^{(4)}(z)=z^{10}, \\
& Q_{5}^{(4)}(z)=z^{6}\left(z^{9}-2822400\right), \\
& Q_{6}^{(4)}(z)=z^{3}\left(z^{18}-31046400 z^{9}-12517908480000\right) .
\end{aligned}
$$

\section{4. ЗАКЛЮЧЕНИЕ}

В данной работе изучались семейства специальных полиномов, связанные с рациональными решениями иерархии второго уравнения Пенлеве. Был найден ряд рекуррентных соотношений, которому удовлетворяют эти полиномы. Отметим, что некоторые из полученных соотношений справедливы для любого семейства. Это еще раз подчеркивает общность свойств полиномов Яблонского-Воробьева, соответствующих различным уравнениям иерархии. Также было найдено дифференциальноразностное соотношение, позволяющее последовательно строить полиномы.

Полиномы Яблонского-Воробьева с точностью до нормировки совпадают с полиномиальными $\tau$-функциями $\tau_{n}^{(N)}(z)$ рациональных решений. Это означает, что соотношение (2.20) легко преобразуется в соотношение для $\tau_{n}^{(N)}(z)$, которое при $N=1$ есть уравнение Тоды. Следовательно, эти соотношения при $N>1$ можно рассматривать как аналоги уравнений для цепочки Тоды [17], [18].

3 Теоретическая и математическая физика, т. 153, № 1, 2007 г. 


\section{ПРИЛОЖЕНИЕ}

Рациональные решения уравнения $P_{2}^{(N)}$ могут быть найдены с помощью формул (1.4) и (2.20). Примеры приведены ниже.

$$
\begin{aligned}
& P_{2} \equiv P_{2}^{(1)}: w_{z z}-2 w^{3}-z w-\alpha=0 ; \\
& w(z ; 1)=-\frac{1}{z} \text {, } \\
& w(z ; 2)=-\frac{2\left(z^{3}-2\right)}{z\left(z^{3}+4\right)}, \\
& w(z ; 3)=-\frac{3 z^{2}\left(z^{6}+8 z^{3}+160\right)}{\left(z^{3}+4\right)\left(z^{6}+20 z^{3}-80\right)}, \\
& w(z ; 4)=-\frac{4\left(z^{15}+50 z^{12}+1000 z^{9}-22400 z^{6}-112000 z^{3}-224000\right)}{z\left(z^{9}+60 z^{6}+11200\right)\left(z^{6}+20 z^{3}-80\right)} . \\
& P_{2}^{(2)}: w_{z z z z}-10 w^{2} w_{z z}-10 w w_{z}^{2}+6 w^{5}-z w-\alpha=0 ; \\
& w(z ; 1)=-\frac{1}{z}, \\
& w(z ; 2)=-\frac{2}{z}, \\
& w(z ; 3)=-\frac{3\left(z^{5}+96\right)}{z\left(z^{5}-144\right)}, \\
& w(z ; 4)=-\frac{4\left(z^{15}-72 z^{10}+217728 z^{5}-1741824\right)}{z\left(z^{5}-144\right)\left(z^{10}-1008 z^{5}-48384\right)}, \\
& w(z ; 5)=-\frac{5 z^{4}\left(z^{20}-2016 z^{15}+6967296 z^{10}+97542144 z^{5}+294967443456\right)}{\left(z^{15}-4032 z^{10}-3048192 z^{5}+146313216\right)\left(z^{10}-1008 z^{5}-48384\right)} . \\
& P_{2}^{(3)}: w_{z z z z z z}-14 w^{2} w_{z z z z}-56 w w_{z} w_{z z z}-42 w\left(w_{z z}\right)^{2}-70\left(w_{z}\right)^{2} w_{z z}+ \\
& +70 w^{4} w_{z z}+140 w^{3}\left(w_{z}\right)^{2}-20 w^{7}-z w-\alpha=0 ; \\
& w(z ; 1)=-\frac{1}{z} \\
& w(z ; 2)=-\frac{2}{z}, \\
& w(z ; 3)=-\frac{3}{z}, \\
& w(z ; 4)=-\frac{4\left(z^{7}-5400\right)}{z\left(z^{7}+7200\right)}, \\
& w(z ; 5)=-\frac{5\left(z^{21}-8640 z^{14}+634521600 z^{7}+268738560000\right)}{z\left(z^{14}+64800 z^{7}-93312000\right)\left(z^{7}+7200\right)} . \\
& P_{2}^{(4)}: w_{z z z z z z z z}-18 w^{2} w_{z z z z z z}-108 w w_{z} w_{z z z z z}+ \\
& +\left(126 w^{4}-228 w w_{z z}-210\left(w_{z}\right)^{2}\right) w_{z z z z}-138 w\left(w_{z z z}\right)^{2}- \\
& -\left(756 w_{z} w_{z z}-1008 w^{3} w_{z}\right) w_{z z z}-182\left(w_{z z}\right)^{3}+756 w^{3}\left(w_{z z}\right)^{2}+ \\
& +\left(3108 w^{2}\left(w_{z}\right)^{2}-420 w^{6}\right) w_{z z}+798 w\left(w_{z}\right)^{4}-1260 w^{5}\left(w_{z}\right)^{2}+ \\
& +70 w^{9}-z w-\alpha=0 \text {; }
\end{aligned}
$$




$$
\begin{aligned}
& w(z ; 1)=-\frac{1}{z}, \\
& w(z ; 2)=-\frac{2}{z}, \\
& w(z ; 3)=-\frac{3}{z}, \\
& w(z ; 4)=-\frac{4}{z}, \\
& w(z ; 5)=\frac{-5\left(z^{9}+2257920\right)}{z\left(z^{9}-2822400\right)} .
\end{aligned}
$$

\section{Список литературы}

[1] М. Абловиц, Х. Сигур, Солитонъ и метод обратной задачи, Мир, М., 1987.

[2] А. Ньюэлл, Солитоны в математике и физике, Мир, М., 1989.

[3] Н.А. Кудряшов, Аналитическая теория нелинейных дифференциальных уравнений, Ин-т компьютерных исследований, Москва-Ижевск, 2004.

[4] А. И. Яблонский, Вести АН БССР. Сер. физ.-тех. наук, 3 (1959), 30-35.

[5] А. П. Воробьев, Дифферени. уравнения, 1 (1965), 79-81.

[6] P. A. Clarkson, Phys. Lett. A, 319:1-2 (2003), 137-144.

[7] K. Okamoto, Math. Ann., 275:2 (1986), 221-255.

[8] H. Umemura, Sugaki Expositions, 11:1 (1998), 77-100.

[9] N. A. Kudryashov, Phys. Lett. A, 224:6 (1997), 353-360.

[10] P. A. Clarkson, E. L. Mansfield, Nonlinearity, 16:3 (2003), R1-R26.

[11] M. V. Demina, N. A. Kudryashov, Chaos, Solitons \& Fractals, 32:2 (2007), 526-537.

[12] N.A. Kudryashov, A. Pickering, "Rational and special solutions of the $P_{I I}$ hierarchy", SIDE III - Symmetries and Integrability of Difference Equations (Sabaudia, 1998), CRM Proc. Lecture Notes, 25, eds. D. Levi, O. Ragnisco, AMS, Providence, RI, 2000, 245-253.

[13] V.I. Gromak, I. Laine, S. Shimomura, Painlevé Differential Equations in the Complex Plane, de Gruyter Stud. Math., 28, de Gruyter, Berlin-New York, 2002.

[14] N. A. Kudryashov, M. B. Soukharev, Phys. Lett. A, 237:4-5 (1998), 206-216.

[15] H. Airault, Stud. Appl. Math., 61:1 (1979), 31-53.

[16] A. N. W. Hone, Phys. D, 118:1-2 (1998), 1-16.

[17] В. Э. Адлер, А. Б. Шабат, Р. И. Ямилов, ТМФ, 125:3 (2000), 355-424.

[18] В. Г. Марихин, А. Б. Шабат, ТМФ, 118:2 (1999), 217-228.

Поступила в редакцию 27.12.2006 